Article

\title{
Long-Term Fertilization Affects Soil Microbiota, Improves Yield and Benefits Soil
}

\author{
Felix R. Kurzemann ${ }^{1,2, *,+}$ (), Ulrich Plieger ${ }^{1, \dagger}{ }^{+}$, Maraike Probst ${ }^{1}$, Heide Spiegel ${ }^{3}{ }^{(0)}$, \\ Taru Sandén ${ }^{3} \mathbb{D}$, Margarita Ros ${ }^{4} \mathbb{D}$ and Heribert Insam ${ }^{1}$ (D) \\ 1 Department of Microbiology, University Innsbruck, 6020 Innsbruck, Austria; \\ ulrich.plieger@student.uibk.ac.at (U.P.); maraike.probst@uibk.ac.at (M.P.); heribert.insam@uibk.ac.at (H.I.) \\ 2 Management Center Innsbruck, 6020 Innsbruck, Austria \\ 3 Department for Soil Health and Plant Nutrition, Austrian Agency for Health and Food Safety, 1220 Vienna, \\ Austria; adelheid.spiegel@ages.at (H.S.); taru.sanden@ages.at (T.S.) \\ 4 Centro de Edafología y Biología Aplicada del Segura (CEBAS-CSIC), 30100 Murcia, Spain; \\ margaros@cebas.csic.es \\ * Correspondence: felix.kurzemann@student.uibk.ac.at \\ + Equal contribution as first author.
}

Received: 27 August 2020; Accepted: 26 October 2020; Published: 28 October 2020

\begin{abstract}
Fertilization of soil is needed to fulfill the growing demand for livestock feed and human food requirements. However, fertilization has short and long-term impacts on the soil microbiota. These, in turn, may influence plant viability and growth. We investigated the soil microbiota of a 27-year field trial, focusing on the influences of mineral nitrogen $(\mathrm{N})$ fertilization, different composts and combinations of compost plus mineral $\mathrm{N}$ as soil amendments. Two $\mathrm{N}$ rates $(0$ and $80 \mathrm{~kg}$ per ha) and four different composts (urban organic waste compost (OWC) green waste compost (GC), farmyard manure (MC) compost and sewage sludge compost (SSC)) were used. Soil samples for this study were taken in 2018 after the growing season of maize. In addition to maize yield, the effects on soil physicochemical properties and the soil microbiota were analyzed. There was a trend for increased maize yields for all fertilizers; however, only the application of GC and SSC in combination with mineral $\mathrm{N}$ fertilizer showed significant effects. The different organic amendments influenced physicochemical soil properties. Phosphorus concentrations were three times higher in plots receiving SSC $\left(\approx 312 \mathrm{mg} \mathrm{kg}^{-1}\right)$ and SSC $+\mathrm{N}\left(\approx 297 \mathrm{mg} \mathrm{kg}^{-1}\right)$ than control $\left(\approx 89 \mathrm{mg} \mathrm{kg}^{-1}\right)$ or mineral $\mathrm{N}$ fertilizer $\left(\approx 97 \mathrm{mg} \mathrm{kg}^{-1}\right)$ alone. Magnesium concentrations in plots treated with SSC $\left(\approx 74 \mathrm{mg} \mathrm{kg}^{-1}\right.$ ) were lower compared to soils treated with GC and MC, respectively $\left(\approx 135 \mathrm{mg} \mathrm{kg}^{-1}\right.$ and $126 \mathrm{mg} \mathrm{kg}^{-1}$ ). Bacteria exceeded the fungal community in terms of both richness and diversity. While the bacterial community composition differed significantly among the treatments, the fungal community composition was rather unaffected. Our conclusion is that composts produced from various substrates serve as valuable nutrient sources for plants and can partially substitute mineral $\mathrm{N}$. In addition, composts increased soil microbial biomass and modulated the composition of the soil's microbial community.
\end{abstract}

Keywords: composts; mineral fertilizers; organic fertilizers; microbial diversity; microbial biomass; field experiment

\section{Introduction}

Recycling of agricultural, domestic and industrial organic wastes by composting provides a better environmental solution compared to landfilling or incineration [1-3]. Usually composts are free from human pathogens and weed seeds [4], and their use as soil organic amendments improves soil quality 
by increasing its organic carbon (C) and nutrient contents [2]. The combined use of organic and mineral fertilizers has been described as an effective approach to further increase soil fertility and crop yields compared to applications of only organic or mineral fertilizers [5].

In addition to the use of organic fertilizers, crop rotation systems can enhance $\mathrm{N}$ availability in agricultural systems, increase soil microbial diversity and improve productivity. Shafi et al. [6] showed that crop rotation systems enhance the amount of nitrogen $(\mathrm{N})$ available in soil, thereby reducing the requirement of fertilization while still promoting plant growth and health [7]. Soman et al. [8] showed that crop rotation in combination with long-term fertilization led to increased microbial diversity in the maize rhizosphere. They found increased abundances of Acidobacteria, Actinobacteria and Proteobacteria in fields where crop rotation was practiced compared to monocultural systems. Therefore, the combination of crop rotation with composts seems a valuable strategy to substitute mineral fertilizers [8].

Many of the above-mentioned effects are ascribed to allochthonous and autochthonous microorganisms that are supplied and/or stimulated by the addition of compost, respectively. As compost properties heavily depend on the substrate material, compost is a rather heterogenic material. Municipal wastes show often relatively high $\mathrm{N}$ contents; gardening waste composts are rich in bulking agents (e.g., branches); compost from sewage sludge is mainly composed of microbial biomass and contains high amounts of phosphorus $(\mathrm{P})$; and many manure composts contain comparatively high magnesium $(\mathrm{Mg})$ contents. As the environment shapes the microbiome, both substrate and compost heterogeneity are reflected in the composition of the compost microbial community [9-12]. Another key factor influencing the soil microbiome is plant growth. Maize is known to host specific bacterial and archaeal communities, as shown by Chelius et al. [13]. They detected mainly Proteobacteria, one example being diazotrophic Burkholderia, and also Gram-positive bacteria such as Cytophagales and Bacillales.

Microorganisms in the soil fulfill important ecological functions, such as nutrient cycling, organic matter decomposition and formation of soil aggregates, which are key factors for soil health, and furthermore support plant growth and yield [14]. High diversity and abundance of microorganisms are considered beneficial for soil and for increasing the stability of the soil's ecosystem [15]. The soil microbiome can be investigated best by sequencing of microbial marker genes, from which the taxonomic composition of the microbial soil community can be inferred [16]. In addition, soil enzymatic activities are a measure for microbial activity, which has been shown as a useful indicator of soil fertility [17].

In this study, the long-term effects of mineral $\mathrm{N}$ fertilizer, and different composts alone and in combination with mineral $\mathrm{N}$ fertilizer, on the soil, especially the soil microbiome, were investigated. The soil of the plots has been studied by different authors for $15[18,19]$ and $25[2,20]$ years, respectively. The previous studies showed the beneficial effect of compost application. In addition to higher plant yields, higher contents of soil organic matter $(\mathrm{SOM})$ and total organic carbon $\left(\mathrm{C}_{\text {org }}\right)$ and $\mathrm{N}$ were reported $[2,19]$. Long-term fertilization with composts also enhanced the microbial biomass $\left(C_{\text {mic }}\right)$ and enzyme activities compared to soils treated with mineral $\mathrm{N}$ fertilizers or without any $\mathrm{N}$ fertilization. While previous studies detected a higher diversity of bacteria in compost-treated soils compared to the unfertilized soil and soil amended solely with mineral $\mathrm{N}$ fertilizers [18], methodological limitations hampered detailed investigation of the soil microbiome. Therefore, we investigated the microbial diversity with NGS (next generation sequencing), to get a closer look at the differences in the microbial community after long-term fertilization.

We hypothesize that long-term organic amendments (i) increase soil microbial diversity, (ii) increase soil microbial activity, (iii) alter the microbial community depending on the type of compost applied and (iv) improve crop yields over soil solely receiving mineral $\mathrm{N}$ fertilization. 


\section{Materials and Methods}

\subsection{Experimental Design, Soil Sampling and Crop Yield}

In 1991, a crop rotation experiment with maize (Zea mays), spring wheat (Triticum aestivum) and winter barley (Hordeum vulgare) was implemented at Ritzlhof ( $48^{\circ} 18^{\prime} \mathrm{N} 14^{\circ} 25^{\prime} \mathrm{E}$ ), close to Linz in Upper Austria. In 2004, peas (Pisum sativum) were included in the rotation, followed by winter wheat (Triticum aestivum) (Table S1).

The experimental setup was a completely randomized plot experiment with 40 plots of $30 \mathrm{~m}^{2}$ (10 treatments with 4 replicates) in a two-factorial design. Soils were either amended with mineral nitrogen fertilizer at a rate of $80 \mathrm{~kg} \mathrm{~N} \mathrm{ha}^{-1}$ as $\mathrm{NH}_{4} \mathrm{NO}_{3}(80 \mathrm{~kg} \mathrm{~N})$ or without nitrogen (control = $\left.0 \mathrm{~kg} \mathrm{~N}\right)$. As a second factor, $175 \mathrm{~kg} \mathrm{~N}$ ha $^{-1}$ was applied via different composts (urban organic waste compost (OWC), green waste compost (GC), farmyard manure compost (MC) and sewage sludge compost (SSC)) without or with $80 \mathrm{~kg} \mathrm{~N} \mathrm{ha}^{-1}$ of mineral fertilizer $((\mathrm{OWC}+\mathrm{N}),(\mathrm{GC}+\mathrm{N}),(\mathrm{MC}+\mathrm{N}),(\mathrm{SSC}+\mathrm{N}))$. Soils without compost application received $75 \mathrm{~kg} \mathrm{P}_{2} \mathrm{O}_{5} \mathrm{ha}^{-1}$ and $150 \mathrm{~kg} \mathrm{~K}_{2} \mathrm{O} \mathrm{ha}^{-1}$ per year.

Mineral and organic fertilizers were applied annually since 1991, either in spring (for spring wheat and maize) or in autumn (for winter barley and winter wheat). Exceptions were, however, 2004, 2008, 2010, 2012, 2014 and 2017 when no fertilizers of any kind were applied. Compost was incorporated into the $15 \mathrm{~cm}$ soil layer with a rotary harrow followed by sowing [2].

The characteristics of the composts are given in Table 1. According to the Austrian compost ordinance, $\mathrm{MC}$ and $\mathrm{OWC}$ are "A+" (best category), qualifying these materials for organic agriculture; GC is classified as " $\mathrm{A}$ " class compost; and SSC is classified as "quality sewage sludge compost." The latter two compost types are approved for conventional but not organic agriculture.

Table 1. Physicochemical properties of the four different composts (compost sampled in 2018).

\begin{tabular}{ccccc}
\hline Compost & OWC & GC & MC & SSC \\
\hline $\mathrm{pH}$ & 7.85 & 7.26 & 8.15 & 7.1 \\
$\mathrm{~N}_{\text {tot }}\left(\mathrm{g} \mathrm{kg}^{-1}\right)$ & 10.5 & 6.6 & 5.9 & 14.0 \\
$\left.\mathrm{Corg}_{\mathrm{rg} \mathrm{kg}}{ }^{-1}\right)$ & 225 & 116 & 183 & 231 \\
$\mathrm{C} / \mathrm{N}$ & 21.4 & 17.6 & 31.0 & 16.5 \\
$\mathrm{P}\left(\mathrm{g} \mathrm{kg}^{-1}\right)$ & 2.0 & 1.6 & 1.9 & 9.7 \\
$\mathrm{~K}\left(\mathrm{~g} \mathrm{~kg}^{-1}\right)$ & 4.8 & 5.5 & 7.8 & 9.1 \\
$\mathrm{Cu}\left(\mathrm{mg} \mathrm{kg}^{-1}\right)$ & 101 & 33 & 24 & 67 \\
$\mathrm{Zn}\left(\mathrm{mg} \mathrm{kg}^{-1}\right)$ & 233 & 118 & 155 & 260 \\
$\mathrm{Ni}\left(\mathrm{mg} \mathrm{kg}^{-1}\right)$ & 21.5 & 28.6 & 23.6 & 59.0 \\
$\mathrm{Cr}\left(\mathrm{mg} \mathrm{kg}^{-1}\right)$ & 39.5 & 50.5 & 53.1 & 27.0 \\
$\mathrm{~Pb}\left(\mathrm{mg} \mathrm{kg}^{-1}\right)$ & 29.6 & 23.1 & $<\mathrm{LOD}$ & 23.0 \\
$\mathrm{Cd}\left(\mathrm{mg} \mathrm{kg}^{-1}\right)$ & 0.50 & 0.20 & 0.30 & 0.47 \\
$\mathrm{Hg}\left(\mathrm{mg} \mathrm{kg}^{-1}\right)$ & 0.10 & 0.09 & 0.04 & 0.20 \\
\hline
\end{tabular}

Data are on dry mass basis. LOD = limit of detection. Urban organic waste compost (OWC), green waste compost (GC), farmyard manure compost (MC) and sewage sludge compost (SSC).

Maize was harvested in September 2018 with a plot harvester $\left(8.4 \mathrm{~m}^{2}\right.$ the central area of every plot), and grain yield (normalized for $14 \%$ water content) was determined by weighing.

Ten soil samples of each of the four replicate plots from 0-25 cm depth were taken with an auger (30 $\mathrm{mm}$ in diameter) after harvest of maize in autumn 2018. The samples of each plot were pooled, sieved (mesh size $2 \mathrm{~mm}$ ) and frozen in plastic bags at $-20^{\circ} \mathrm{C}$ until analysis.

\subsection{Soil Physicochemical Properties}

The $\mathrm{pH}$ of the soil was measured electrochemically $(\mathrm{pH} / \mathrm{mV}$ Pocket Meter $\mathrm{pH} 340 \mathrm{i}$, WTW, Weilheim, Germany) in $0.01 \mathrm{M} \mathrm{CaCl}_{2}(1: 5 w / v)$ according to ÖNORM L1083 [21]. The electric conductivity (EC) $(1: 5 w / v)$ was measured with a conductivity meter (LF 330, WTW, Weilheim, Germany). Total organic 
C $\left(\mathrm{C}_{\text {org }}\right.$ ) was analyzed by dry combustion with a LECO RC-612TruMac CN (Leco, Geleen, The Netherlands) at $650{ }^{\circ} \mathrm{C}$ according to ÖNORM L1080 [22]. Soil organic matter (SOM) was measured through weight loss after ignition in a muffle furnace (CWF 1000, Carbolite, Lilienthal, Germany) for $5 \mathrm{~h}$ at $550{ }^{\circ} \mathrm{C}$. Total $\mathrm{N}\left(\mathrm{N}_{\text {tot }}\right)$ concentration was determined according to ÖNORM EN16168 [23] via elemental analysis using a CNS 2000 SGA-410-06 (Leco, Geleen, The Netherlands) at $1250{ }^{\circ} \mathrm{C}$. The labile soil $\mathrm{C}$ fraction was analyzed using potassium permanganate $\left(0.02 \mathrm{M} \mathrm{KMnO}_{4}\right)$ followed by a titration of the unconsumed $\mathrm{KMnO}_{4}$ solution with sodium oxalate $\left(\mathrm{Na}_{2} \mathrm{C}_{2} \mathrm{O}_{4}\right)$ to determine the active $\mathrm{C}$ (AC) pool [24]. Heavy metal (HM) concentrations were analyzed after digestion of $1 \mathrm{~g}$ soil with $10 \mathrm{~mL}$ inverse aqua regia in a microwave (speedwave 4, Berghof Products + Instruments, Eningen, Germany) via ICP-OES spectrophotometry (Genesis, Spectro, Kleve, Germany) according to DIN EN 13,346 [25].

Soil dry matter (DM) and soil water holding capacity (WHC) were measured according to Öhlinger [26,27]. The amount of $\mathrm{NH}_{4}{ }^{+}$and the $\mathrm{N}$ mineralization potential (ammonification) under waterlogged conditions were tested according to Kandeler [28,29]. For the ammonium determination, $15 \mathrm{~g}$ soil and $60 \mathrm{~mL} \mathrm{CaCl}{ }_{2}$-solution $(0.025 \mathrm{M})$ were used. Magnesium $(\mathrm{Mg})$ was extracted with a $0.0125 \mathrm{M} \mathrm{CaCl}_{2}$ solution according to the method of Schachtschabel (ÖNORM L1093 [30]) and analyzed using a flame atomic absorption spectrometer (Thermo Fisher iCE 3500 (Thermo Scientific, Waltham, $\mathrm{MA}, \mathrm{USA})$ ). The exchangeable cations $\mathrm{Mg}\left(\mathrm{Mg}_{\text {excha }}\right), \mathrm{Ca}\left(\mathrm{Ca}_{\text {excha }}\right), \mathrm{Na}\left(\mathrm{Na}_{\text {excha }}\right)$ and $\mathrm{K}\left(\mathrm{K}_{\text {excha }}\right)$ and the effective CEC were analyzed in unbuffered $0.1 \mathrm{M} \mathrm{BaCl}_{2}$ solution in a ratio of 1:20 with $\mathrm{H}_{2} \mathrm{O}$ according to ÖNORM L1086-1 [31]. Potassium (K) and phosphorus (P) were determined using the calcium-acetate-lactate (CAL) extraction with a spectral photometer $(\mathrm{P})$ using the molybdenum blue method and a flame photometer (K), using a segmented flow analyzer (SAN, Skalar Analytical BV, Breda, The Netherlands) [20].

\subsection{Soil Microbial Biomass and Enzyme Activities}

Soil microbial biomass was determined with the substrate-induced-respiration method using an EGA61-Soil Respiration Device (ADC BioScientific, Hoddesdon, UK). Enzymatic activities were measured as reported before $[18,19]$. Dehydrogenase activity was determined by reducing INT to INTF. Urease activity was measured via buffered urease reaction. Alkaline phosphatase and $\beta$-glucosidase activities were measured according to Tabatabai et al. [32] and Eivazi and Tabatabai [33]. For the alkaline phosphatase assay, $0.5 \mathrm{~g}$ soil and $2 \mathrm{~mL}$ modified universal buffer (MUB) at $\mathrm{pH} 11$ and p-nitrophenyl phosphatase were used as substrate, while for determining the $\beta$-glucosidase activity, $0.5 \mathrm{~g}$ soil and $2 \mathrm{~mL}$ MUB at $\mathrm{pH} 6.5$ and p-nitrophenyl- $\beta-\mathrm{D}$-glucopiranoside were used as substrate. After incubation, $0.5 \mathrm{~mL}$ of $0.5 \mathrm{M} \mathrm{CaCl}_{2}$ was added, followed by the addition of two milliliters of $0.1 \mathrm{M}$ Tris (hydroxymethyl) aminomethane sodium hydroxide ( $\mathrm{pH} 12$ ). For the alkaline phosphatase assay, $2 \mathrm{~mL} 0.5 \mathrm{M} \mathrm{NaOH}$ were added instead. After vortexing, resulting p-nitrophenol was measured spectrophotometrically at $400 \mathrm{~nm}$. All photometric enzyme activity measurements were conducted with a photometer (Helios Alpha100-240, Thermo Electron, Gloucester, UK).

\subsection{Soil DNA Extraction and Sequencing}

Soil DNA was extracted with the NucleoSpin ${ }^{\circledR}$ soil kit (Macherey-Nagel, Düren, Germany) according to the user manual. MicroSynth (Balgach, Switzerland) carried out the Illumnia amplicon sequencing of microbiomes using universal $16 \mathrm{~S}$ primer for detection of bacteria and archaea (515f: GTG CCA GCM GCC GCG GTA; 806r: GGA CTA CHV GGG TWT CTA AT) and ITS primer for fungi (ITS3: GCA TCG ATG AAG AAC GCA GC; ITS4: TCC TCC GCT TAT TGA TAT GC). Resulting sequencing data were processed with the CoMa pipeline [34]. The process was the same for fungi, bacteria and archaea. Sequences of all samples were trimmed (fungi: forward side $26 \mathrm{bp}$, reverse side $25 \mathrm{bp}$; bacteria and archaea: forward side $21 \mathrm{bp}$, reverse side 20) and a minimum quality score of 30 was chosen for sequences to be retained. Reads were merged and sequences with lengths of $275-474 \mathrm{bp}$ were selected for fungi, whereas a length of 240-270 bp was the defined range for bacteria and archaea. Operational taxonomic units (OTUs) were clustered at a $97 \%$ similarity level, and both SILVA (release 
132) (alignment of the 16S small subunit ribosomal RNA) and Greengenes (release 13_5) served as reference databases for taxonomic assignment. Rare OTUs with less than four occurrences among all samples were omitted from further analysis. Only OTUs present in at least two out of four replicate plots were considered for further analysis.

\subsection{Statistical Analyses}

For statistical analyses, R [35] and relevant packages therein were used. For univariate analysis, the statistical difference between treatments was assessed using the Kruskal-Wallis test or analysis of variance (ANOVA), depending on data distribution and normality assumption. For all analyses, a confidence interval of $95 \%$ was applied. Principal component analysis (PCA) was used to compress the multidimensional character of the physicochemical data into fewer variables (components). Variables subjected to PCA were ammonification rate; concentrations of $\mathrm{NH}_{4}, \mathrm{Mg}$ and $\mathrm{K}$; infrared gas analyzer (IRGA) measurements (basal respiration, metabolic quotient and $\mathrm{C}_{\text {mic }}$ ); contents of $\mathrm{DM}, \mathrm{C}_{\text {org }}, \mathrm{N}$ mineral potential and $\mathrm{AC}$; $\mathrm{pH}$; EC; WHC; and the exchange capacities of $\mathrm{Ca}$ and $\mathrm{Na}$. Prior to component extraction, data were scaled and centered. Varimax rotation was applied to obtain orthogonal components. In order to assess the influences of the original variables on the resulting clustering of sample groups, original variables (scaled and centered) were correlated with the components using Pearson correlation. Microbial richness for treatment groups was estimated using the R package "Breakaway" [36]. Microbial units overrepresented in treatment groups were analyzed using "ALDEx2" [37]. Principal coordinate analysis (PCoA) was used to visualize differences and similarities between the samples' microbial compositions. The distances between samples were calculated on a binary matrix using the Jaccard distance. The differences between treatments were calculated using permutation (999 permutations, Adonis function of the R package "Vegan"). Venn diagrams were calculated and visualized using the R package "Vennerable" [38]. The difference between the total sum of OTUs of a sample group and its estimated richness results from the Venn diagram not considering the rare biosphere (i.e., small OTUs and OTUs not detected in all individual samples) of a sample group. This rare biosphere is excluded from the Venn analysis to ensure an adequate representativeness. Bar charts illustrating microbial compositions were generated using the R package “Compositions" [39]. Data were visualized using R and Microsoft Excel.

\section{Results}

\subsection{Crop Yield}

Addition of organic and mineral $\mathrm{N}$ fertilization increased the maize yield by $6-14 \%$ compared to the control treatment (Figure 1). Treatment SSC $+\mathrm{N}\left(\mathrm{p}_{\text {Wilcox }}=0.029\right)$ and GC $+\mathrm{N}\left(\mathrm{p}_{\text {Wilcox }}=0.057\right)$ showed the highest maize yield (Figure 1); however, a statistically significant difference was only found compared to the control. 


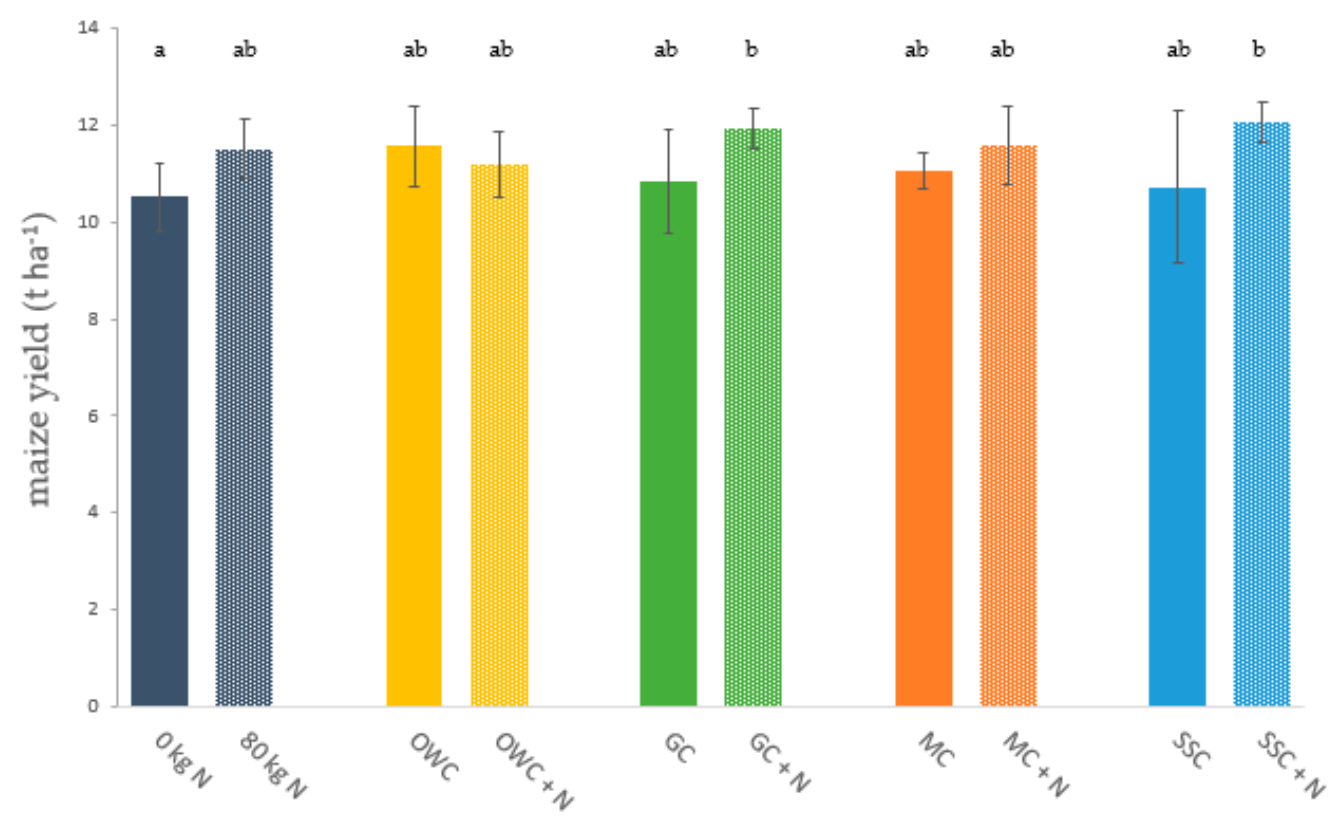

Figure 1. Maize yield (mean $\pm \mathrm{SD} ; n=4)$. Columns with the same lowercases letters are not significantly different $(p \leq 0.05)$ between treatments. Control $(0 \mathrm{~kg} \mathrm{~N}), 80 \mathrm{~kg}$ mineral N ha ${ }^{-1}(80 \mathrm{~kg} \mathrm{~N})$, urban organic waste compost (OWC), green waste compost (GC), farm yard manure compost (MC), sewage sludge compost (SSC), $+\mathrm{N}$ application of $80 \mathrm{~kg} \mathrm{~N} \mathrm{ha}^{-1}$.

\subsection{Soil Properties}

We tested for significant differences in soil physicochemical properties between the plots receiving no fertilization, mineral fertilizer, organic composts and plots receiving organic composts and mineral fertilizer. According to multivariate analysis, $40 \%$ of variance in soil properties among the samples was explained by the factor treatment. Principal component analysis representing the physicochemical soil properties of the different treatments clustered the soil samples into three major groups, (i) control and $80 \mathrm{~kg} \mathrm{~N}$ treatment, (ii) SSC and (iii) a joint group of OWC, GC and MC (Figure S1). Differences between compost and compost $+\mathrm{N}$ were mainly not detected or inconsistent. On a Euclidean distance matrix, the addition of $80 \mathrm{~N}$ did not affect soil properties $\left(R_{\text {Adonis }}=0.024\right.$, $p_{\text {Adonis }}=0.147$ ).

The physicochemical parameters showed significant differences among treatments (Table 2). Treatment SCC $+\mathrm{N}$ had significantly higher ECs than soils amended with other treatments, except for the control and soils treated with GC and SSC. Soils amended with organic fertilizers had significantly higher $\mathrm{pH}$ values than soils treated with $80 \mathrm{~kg} \mathrm{~N}$ alone, except for control, $\mathrm{MC}, \mathrm{MC}+\mathrm{N}$ and GC $+\mathrm{N}$. $\mathrm{C}_{\text {org }}$ was higher in organic treatments (OWC, GC, MC, SSC) compared to $0 \mathrm{~kg} \mathrm{~N}$ and $80 \mathrm{~kg}$ N; however, only GC + N, SSC and SSC + N were significantly higher. Treatment SSC followed by GC-amended soils exhibited the highest values for $\mathrm{C}_{\text {org }}$. The soils amended with $80 \mathrm{~kg} \mathrm{~N}$ showed significantly lower SOM values compared to the compost treatments, independent from the addition of N. Soil dry matter (DM) was not significant different among treatments. Active C (AC) was significantly different between GC and SSC $+\mathrm{N}$ treated soils. The amount of $\mathrm{N}_{\text {tot }}$ was higher in soils amended with organic fertilizers compared to $80 \mathrm{~kg} \mathrm{~N}$-amended soil. Nevertheless, soils amended with MC, MC $+\mathrm{N}$ and $\mathrm{OWC}+\mathrm{N}$ were not significantly higher. Compared to the control, in SSC, SSC + N and GC + N-treated soils, significantly higher amounts of $\mathrm{N}_{\text {tot }}$ were found. The rate of ammonification was significantly different between soils treated with SSC and SSC $+\mathrm{N}$. The amount of $\mathrm{NH}_{4}$ showed no significant differences among the treatments. 
Table 2. Physicochemical soil parameters comparing different treatments (mean \pm standard deviation, $n=4$; for treatment, $80 \mathrm{~kg} \mathrm{~N}$ heavy metal contents were analyzed for only one soil sample).

\begin{tabular}{|c|c|c|c|c|c|c|}
\hline Treatment & $\mathrm{EC}\left(\mu \mathrm{S} \mathrm{cm}^{-1}\right)$ & $\mathrm{pH}$ & $C_{\text {org }}\left(\mathrm{g} \mathrm{kg}^{-1}\right)$ & SOM $\left(\mathrm{g} \mathrm{kg}^{-1}\right)$ & DM (\%) & AC (\%) \\
\hline $0 \mathrm{~kg} \mathrm{~N}$ & $36.0 \pm 7.68 \mathrm{ab}$ & $7.09 \pm 0.15 \mathrm{ab}$ & $12.6 \pm 0.12 \mathrm{a}$ & $40.8 \pm 3.49 \mathrm{ab}$ & $84.7 \pm 1.02$ & $4.5 \pm 0.22 \mathrm{ab}$ \\
\hline $80 \mathrm{~kg} \mathrm{~N}$ & $24.0 \pm 2.41 \mathrm{a}$ & $7.00 \pm 0.16 \mathrm{a}$ & $12.6 \pm 0.07 \mathrm{a}$ & $37.6 \pm 0.92 \mathrm{a}$ & $85.5 \pm 0.14$ & $4.33 \pm 0.22 \mathrm{ab}$ \\
\hline OWC & $30.4 \pm 1.75 \mathrm{a}$ & $7.25 \pm 0.07 b c$ & $15.5 \pm 0.79 \mathrm{ab}$ & $44.7 \pm 2.90 \mathrm{bc}$ & $83.6 \pm 1.09$ & $4.36 \pm 0.22 \mathrm{ab}$ \\
\hline GC & $34.0 \pm 3.28 \mathrm{ab}$ & $7.20 \pm 0.08 \mathrm{bc}$ & $15.4 \pm 1.47 \mathrm{ab}$ & $47.5 \pm 3.09 \mathrm{bc}$ & $84.1 \pm 0.45$ & $5.22 \pm 1.43 b$ \\
\hline $\mathrm{MC}$ & $30.7 \pm 4.87 \mathrm{a}$ & $7.14 \pm 0.11 \mathrm{abc}$ & $14.3 \pm 1.46 \mathrm{ab}$ & $50.3 \pm 2.08 \mathrm{bc}$ & $84.6 \pm 0.41$ & $4.28 \pm 0.15 \mathrm{ab}$ \\
\hline SSC & $39.6 \pm 3.94 \mathrm{ab}$ & $7.38 \pm 0.02 c$ & $16.8 \pm 1.54 b$ & $44.5 \pm 2.63 c$ & $83.6 \pm 0.27$ & $4.14 \pm 0.14 \mathrm{ab}$ \\
\hline $\mathrm{OWC}+\mathrm{N}$ & $34.2 \pm 3.95 \mathrm{a}$ & $7.26 \pm 0.09 b c$ & $15.2 \pm 0.27 \mathrm{ab}$ & $48.0 \pm 0.94 b c$ & $84.0 \pm 0.71$ & $4.05 \pm 0.11 \mathrm{ab}$ \\
\hline $\mathrm{GC}+\mathrm{N}$ & $34.0 \pm 6.84 \mathrm{a}$ & $7.23 \pm 0.02 a b c$ & $15.9 \pm 0.93 b$ & $52.2 \pm 1.43 \mathrm{bc}$ & $84.0 \pm 0.42$ & $4.01 \pm 0.11 \mathrm{ab}$ \\
\hline $\mathrm{MC}+\mathrm{N}$ & $35.8 \pm 8.80 \mathrm{a}$ & $7.16 \pm 0.11 \mathrm{abc}$ & $14.7 \pm 1.08 \mathrm{ab}$ & $44.0 \pm 2.43 \mathrm{bc}$ & $84.2 \pm 0.50$ & $3.98 \pm 0.23 \mathrm{ab}$ \\
\hline \multirow[t]{2}{*}{$\mathrm{SSC}+\mathrm{N}$} & $49.4 \pm 10.5 b$ & $7.40 \pm 0.03 c$ & $16.2 \pm 0.69 \mathrm{~b}$ & $44.9 \pm 0.64 \mathrm{bc}$ & $84.4 \pm 0.64$ & $3.82 \pm 0.06 a$ \\
\hline & $\mathrm{N}_{\text {(tot) }}\left(\mathrm{g} \mathrm{kg}^{-1}\right)$ & $\begin{array}{c}\text { Ammonification } \\
\text { Rate } \\
\left(\mu \mathrm{g} \mathrm{Ng}^{-1} \mathrm{DM}\right)\end{array}$ & $\begin{array}{c}\mathrm{NH}_{4} \\
\left(\mu \mathrm{g} \mathrm{N} \mathrm{g} \mathrm{DM}{ }^{-1}\right)\end{array}$ & $\mathrm{Mg}\left(\mathrm{mg} \mathrm{kg}^{-1}\right)$ & $\mathrm{K}\left(\mathrm{mg} \mathrm{kg}^{-1}\right)$ & $\mathrm{P}\left(\mathrm{mg} \mathrm{kg}^{-1}\right)$ \\
\hline $0 \mathrm{~kg} \mathrm{~N}$ & $1.40 \pm 0.03 \mathrm{ab}$ & $1.88 \pm 0.13 \mathrm{ab}$ & $4.47 \pm 0.18$ & $116 \pm 6.1 \mathrm{~b}$ & $121 \pm 20.3 \mathrm{ab}$ & $89.0 \pm 19.3 \mathrm{a}$ \\
\hline $80 \mathrm{~kg} \mathrm{~N}$ & $1.38 \pm 0.12 \mathrm{a}$ & $1.76 \pm 0.42 \mathrm{ab}$ & $4.31 \pm 0.22$ & $119 \pm 7.8 b$ & $191 \pm 7.8 \mathrm{abc}$ & $97.1 \pm 44.9 \mathrm{a}$ \\
\hline OWC & $1.67 \pm 0.07 b c$ & $2.64 \pm 0.41 \mathrm{ab}$ & $4.70 \pm 0.38$ & $104 \pm 14.5 \mathrm{ab}$ & $143 \pm 29.9 \mathrm{abc}$ & $140 \pm 34.3 \mathrm{a}$ \\
\hline GC & $1.65 \pm 0.14 b c$ & $2.66 \pm 0.68 \mathrm{ab}$ & $5.11 \pm 0.73$ & $135 \pm 11.1 b$ & $168 \pm 25.3 \mathrm{abc}$ & $123 \pm 13.6 \mathrm{a}$ \\
\hline $\mathrm{MC}$ & $1.54 \pm 0.12 \mathrm{abc}$ & $2.62 \pm 0.34 \mathrm{ab}$ & $3.52 \pm 1.57$ & $126 \pm 14.6 b$ & $203 \pm 27.5 c$ & $139 \pm 33.9 a$ \\
\hline SSC & $1.74 \pm 0.13 \mathrm{c}$ & $2.81 \pm 0.31 b$ & $4.71 \pm 0.88$ & $73 \pm 6.2 \mathrm{a}$ & $134 \pm 16.3 \mathrm{abc}$ & $312 \pm 56.5 b$ \\
\hline $\mathrm{OWC}+\mathrm{N}$ & $1.63 \pm 0.03 \mathrm{abc}$ & $2.51 \pm 0.49 a b$ & $3.88 \pm 0.80$ & $104 \pm 22.8 \mathrm{ab}$ & $121 \pm 15.4 \mathrm{ab}$ & $149 \pm 48.2 \mathrm{a}$ \\
\hline $\mathrm{GC}+\mathrm{N}$ & $1.74 \pm 0.07 \mathrm{c}$ & $2.45 \pm 0.37 \mathrm{ab}$ & $3.54 \pm 0.10$ & $134 \pm 11.5 b$ & $161 \pm 29.3 a b c$ & $121 \pm 5.0 \mathrm{a}$ \\
\hline $\mathrm{MC}+\mathrm{N}$ & $1.60 \pm 0.09 \mathrm{abc}$ & $2.55 \pm 0.40 a b$ & $3.70 \pm 1.44$ & $129 \pm 19.0 b$ & $199 \pm 35.9 a b c$ & $141 \pm 46.7 a$ \\
\hline \multirow[t]{2}{*}{$\mathrm{SSC}+\mathrm{N}$} & $1.75 \pm 0.06 c$ & $1.54 \pm 0.23 \mathrm{a}$ & $3.82 \pm 0.65$ & $74 \pm 13.2 \mathrm{a}$ & $133 \pm 28.3 a b c$ & $297 \pm 35.9 b$ \\
\hline & $\begin{array}{c}\text { Ca } a_{\text {excha }} \\
\left(\mathrm{cmolc} \mathrm{kg}^{-1}\right)\end{array}$ & $\begin{array}{c}\mathrm{K}_{\text {excha }} \\
\left(\mathrm{cmolc} \mathrm{kg}^{-1}\right)\end{array}$ & $\begin{array}{c}\mathrm{Mg}_{\text {excha }} \\
\left(\mathrm{cmolc} \mathrm{kg}^{-1}\right)\end{array}$ & $\begin{array}{c}\mathrm{Na}_{\text {excha }} \\
\left(\mathrm{cmolc} \mathrm{kg}^{-1}\right)\end{array}$ & WHC (\%) & $\mathrm{Co}\left(\mathrm{mg} \mathrm{kg}^{-1}\right)$ \\
\hline $0 \mathrm{~kg} \mathrm{~N}$ & $14.2 \pm 1.13 \mathrm{ab}$ & $0.42 \pm 0.07$ & $1.43 \pm 0.07 \mathrm{~b}$ & $0.03 \pm 0.00 \mathrm{a}$ & $28.8 \pm 7.52$ & $5.76 \pm 0.23 a$ \\
\hline $80 \mathrm{~kg} \mathrm{~N}$ & $13.0 \pm 0.71 \mathrm{a}$ & $0.42 \pm 0.10$ & $1.40 \pm 0.13 \mathrm{~b}$ & $0.03 \pm 0.00 \mathrm{a}$ & $31.7 \pm 1.06$ & $5.95 \mathrm{ab}$ \\
\hline OWC & $16.0 \pm 1.04 b$ & $0.49 \pm 0.09$ & $1.29 \pm 0.15 \mathrm{ab}$ & $0.03 \pm 0.00 \mathrm{a}$ & $34.6 \pm 2.21$ & $7.50 \pm 1.66 \mathrm{ab}$ \\
\hline GC & $15.0 \pm 0.64 \mathrm{ab}$ & $0.60 \pm 0.09$ & $1.59 \pm 0.13 b$ & $0.03 \pm 0.00 \mathrm{a}$ & $32.9 \pm 1.16$ & $7.65 \pm 0.41 \mathrm{ab}$ \\
\hline $\mathrm{MC}$ & $14.6 \pm 1.26 \mathrm{ab}$ & $0.68 \pm 0.06$ & $1.50 \pm 0.17 b$ & $0.04 \pm 0.00 \mathrm{a}$ & $32.9 \pm 1.46$ & $8.58 \pm 0.83 b$ \\
\hline SSC & $18.8 \pm 0.81 \mathrm{~cd}$ & $0.45 \pm 0.06$ & $0.91 \pm 0.09 \mathrm{a}$ & $0.06 \pm 0.01 \mathrm{~b}$ & $33.5 \pm 1.80$ & $5.54 \pm 1.49 a$ \\
\hline $\mathrm{OWC}+\mathrm{N}$ & $16.3 \pm 1.09 \mathrm{bcd}$ & $0.42 \pm 0.05$ & $1.25 \pm 0.26 \mathrm{ab}$ & $0.03 \pm 0.00 \mathrm{a}$ & $34.0 \pm 2.05$ & $6.66 \pm 0.72 \mathrm{ab}$ \\
\hline $\mathrm{GC}+\mathrm{N}$ & $15.8 \pm 0.31 \mathrm{abd}$ & $0.53 \pm 0.09$ & $1.61 \pm 0.14 b$ & $0.04 \pm 0.01 \mathrm{a}$ & $33.9 \pm 2.52$ & $6.76 \pm 0.66 \mathrm{ab}$ \\
\hline $\mathrm{MC}+\mathrm{N}$ & $14.6 \pm 1.52 \mathrm{ab}$ & $0.64 \pm 0.11$ & $1.49 \pm 0.20 \mathrm{~b}$ & $0.04 \pm 0.01 \mathrm{a}$ & $34.7 \pm 1.84$ & $6.71 \pm 1.00 \mathrm{ab}$ \\
\hline \multirow[t]{2}{*}{$\mathrm{SSC}+\mathrm{N}$} & $18.4 \pm 0.74 \mathrm{~cd}$ & $0.43 \pm 0.09$ & $0.95 \pm 0.15 a$ & $0.07 \pm 0.01 b$ & $31.0 \pm 2.37$ & $6.51 \pm 0.81 \mathrm{ab}$ \\
\hline & $\mathrm{Cr}\left(\mathrm{mg} \mathrm{kg}^{-1}\right)$ & $\mathrm{Cu}\left(\mathrm{mg} \mathrm{kg}^{-1}\right)$ & $\mathrm{Ni}\left(\mathrm{mg} \mathrm{kg}^{-1}\right)$ & $\mathrm{Zn}\left(\mathrm{mg} \mathrm{kg}^{-1}\right)$ & $\mathrm{Cd}\left(\mathrm{mg} \mathrm{kg}^{-1}\right)$ & $\mathrm{Pb}\left(\mathrm{mg} \mathrm{kg}^{-1}\right)$ \\
\hline $0 \mathrm{~kg} \mathrm{~N}$ & $18.5 \pm 1.07$ & $16.91 \pm 1.36$ & $20.91 \pm 0.96$ & $52.6 \pm 2.89$ & $3.70 \pm 0.20$ & $15.45 \pm 0.87$ \\
\hline $80 \mathrm{~kg} \mathrm{~N}$ & $18.05 \pm \mathrm{NA}$ & $15.65 \pm \mathrm{NA}$ & $20.02 \pm \mathrm{NA}$ & $50.05 \pm \mathrm{NA}$ & $3.75 \pm$ NA & $15.35 \pm \mathrm{NA}$ \\
\hline OWC & $19.6 \pm 1.25$ & $18.6 \pm 1.14$ & $22.0 \pm 1.38$ & $61.4 \pm 6.85$ & $4.01 \pm 0.25$ & $17.4 \pm 0.47$ \\
\hline GC & $19.0 \pm 1.57$ & $18.2 \pm 1.65$ & $20.7 \pm 2.59$ & $60.5 \pm 6.65$ & $3.81 \pm 0.16$ & $16.8 \pm 0.77$ \\
\hline MC & $19.7 \pm 1.22$ & $19.9 \pm 3.06$ & $23.3 \pm 5.44$ & $61.9 \pm 2.19$ & $5.25 \pm 2.52$ & $16.9 \pm 0.54$ \\
\hline SSC & $16.5 \pm 5.10$ & $17.7 \pm 5.99$ & $18.6 \pm 6.00$ & $57.5 \pm 18.06$ & $3.39 \pm 1.04$ & $14.0 \pm 4.52$ \\
\hline $\mathrm{OWC}+\mathrm{N}$ & $20.5 \pm 1.10$ & $18.5 \pm 0.81$ & $21.7 \pm 0.83$ & $58.3 \pm 1.92$ & $4.05 \pm 0.11$ & $17.5 \pm 0.35$ \\
\hline $\mathrm{GC}+\mathrm{N}$ & $19.3 \pm 1.27$ & $18.2 \pm 0.95$ & $21.3 \pm 1.26$ & $56.9 \pm 3.56$ & $3.91 \pm 0.27$ & $17.5 \pm 1.11$ \\
\hline $\mathrm{MC}+\mathrm{N}$ & $18.9 \pm 1.35$ & $18.3 \pm 1.01$ & $20.8 \pm 0.88$ & $54.0 \pm 1.37$ & $3.85 \pm 0.13$ & $15.8 \pm 0.50$ \\
\hline $\mathrm{SSC}+\mathrm{N}$ & $19.9 \pm 0.89$ & $21.0 \pm 0.72$ & $21.6 \pm 0.66$ & $63.78 \pm 1.95$ & $3.90 \pm 0.17$ & $16.0 \pm 0.45$ \\
\hline
\end{tabular}

Control $(0 \mathrm{~kg} \mathrm{~N}), 80 \mathrm{~kg}$ mineral $\mathrm{N} \mathrm{ha}^{-1}(80 \mathrm{~kg} \mathrm{~N}) ; 175 \mathrm{~kg} \mathrm{~N}$ ha $^{-1}$ of composts; urban organic waste compost (OWC); green waste compost (GC); farm yard manure compost (MC); sewage sludge compost (SSC); $175 \mathrm{~kg} \mathrm{~N} \mathrm{ha}^{-1}$ of composts $+80 \mathrm{~kg}$ mineral N ; electrical conductivity $(\mathrm{EC})$; total organic carbon $\left(\mathrm{C}_{\mathrm{org}}\right)$; soil organic matter $(\mathrm{SOM})$; dry matter (DM); active $\mathrm{C}(\mathrm{AC})$; total $\mathrm{N}\left(\mathrm{N}_{(\mathrm{tot})}\right)$; ammonium $\left(\mathrm{NH}_{4}{ }^{+}\right)$; magnesium $(\mathrm{Mg})$; potassium $(\mathrm{K})$; phosphorus $(\mathrm{P})$; exchange capacity of $\mathrm{Ca}\left(\mathrm{Ca}_{\text {excha }}\right)$; exchange capacity of $\mathrm{Na}\left(\mathrm{Na}_{\text {excha }}\right)$; exchange capacity of $\mathrm{Mg}\left(\mathrm{Mg}_{\text {excha }}\right)$; exchange capacity of $\mathrm{K}$ ( $\left.\mathrm{K}_{\text {excha }}\right)$; water holding capacity (WHC); cobalt (Co); chromium (Cr); copper (Cu); nickel (Ni); zinc $(\mathrm{Zn})$; cadmium $(\mathrm{Cd})$; lead $(\mathrm{Pb})$. Mean values followed by the same lowercase letters are not significantly different $(p \leq 0.05)$ among the different treatments.

In soils treated with SSC and SSC + N, significantly lower amounts of Mg compared to control, $80 \mathrm{~kg} \mathrm{~N}, \mathrm{GC}, \mathrm{MC}, \mathrm{GC}+\mathrm{N}$ and MC + N treated soils were found. MC-amended soils were highest in K, significantly higher than control and $\mathrm{OWC}+\mathrm{N}$ treated soils. The highest concentration of $\mathrm{P}$ was found in soils amended with SSC, while all other samples had significantly lower amounts. The exchange capacity of $\mathrm{Na}\left(\mathrm{Na}_{\text {excha }}\right)$, was highest in the SCC-amended soils. The control and soils treated with $80 \mathrm{~kg} \mathrm{~N}, \mathrm{OWC}, \mathrm{GC}, \mathrm{MC}$ and MC + N exhibited significantly lower $\mathrm{Ca}_{\text {excha }}$ values than other treatments. Soils amended with SSC and SSC $+\mathrm{N}$ showed the lowest values of $\mathrm{Mg}_{\text {excha }}$, but those of OWC and $\mathrm{OWC}+\mathrm{N}$ were not significantly higher. In this regard, SSC-amended soils were lower than all other 
treatments. $\mathrm{K}_{\text {excha }}$ was not significantly different among treatments. No differences among the control, $80 \mathrm{~kg} \mathrm{~N}$ and organic amendments were observed in the water holding capacity WHC (Table 2).

Fertilization did not significantly affect heavy metal concentrations in the soil, except for cobalt (Co) (Table 2). Cobalt (Co) concentrations were significantly lower in plots without any fertilization, plots treated with SSC and plots treated mineral fertilizer. The addition of MC without mineral fertilizer increased the Co concentration ( $8.6 \mathrm{mg} \mathrm{kg}^{-1}$ vs. $5.5-7.7 \mathrm{mg} \mathrm{kg}^{-1}$ in the other plots).

\subsection{Soil Microbial Biomass and Enzyme Activities}

Soil microbial biomass $\left(\mathrm{C}_{\text {mic }}\right)$ was higher in soils treated with organic amendments; however, not all differences were significant. While OWC, GC and SCC (regardless of the N application) showed similar values for $C_{\text {mic }}\left(410-470 \mu \mathrm{g} \mathrm{C} \mathrm{g}{ }^{-1}\right.$ soil), soils that received $80 \mathrm{~kg} \mathrm{~N}$ ha $^{-1}$ showed significantly lower values ( $360 \mu \mathrm{g} \mathrm{C} \mathrm{g}{ }^{-1}$ soil). The control (400 $\mu \mathrm{g} \mathrm{C} \mathrm{g}{ }^{-1}$ soil) and treatment MC (410 $\mu \mathrm{g} \mathrm{C} \mathrm{g}{ }^{-1}$ soil) showed a significantly lower microbial biomass than soils treated with MC $+\mathrm{N}$ or SSC (Figure 2).

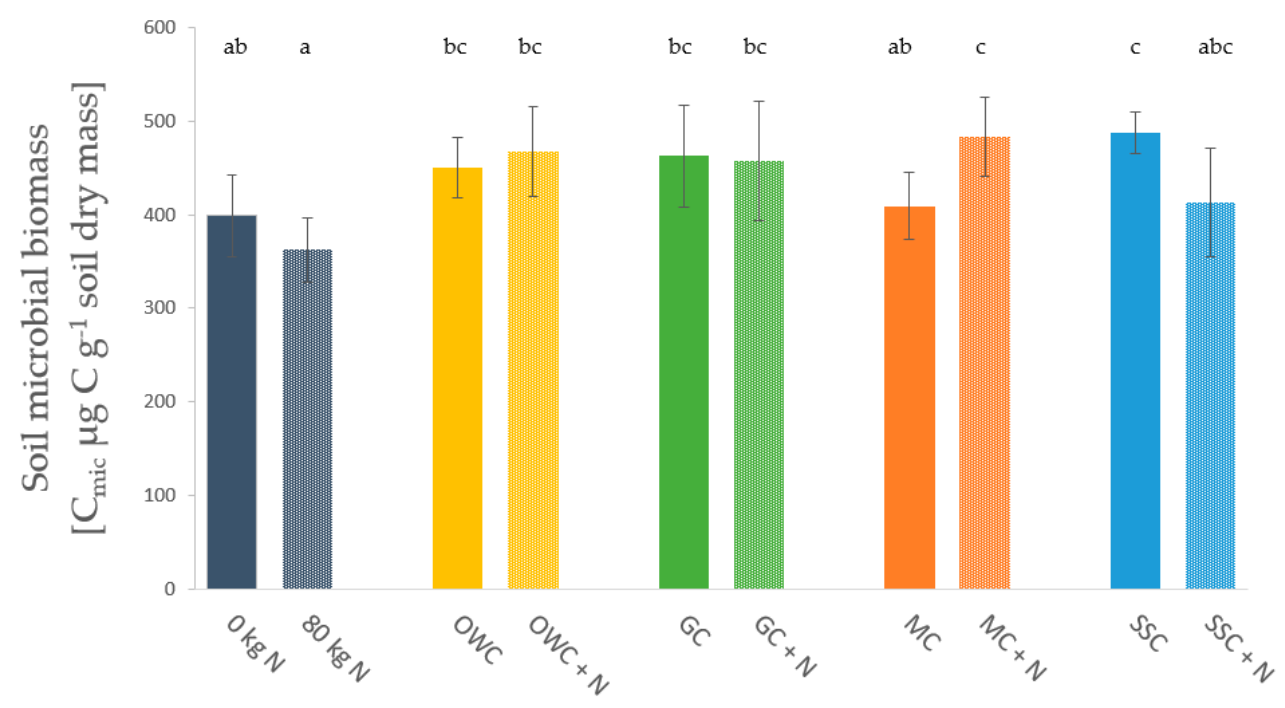

Figure 2. Soil microbial biomass (mean $\pm \mathrm{SD}, n=4$ ). Columns with the same lowercase letters are not significantly different $(p \leq 0.05)$. Control $(0 \mathrm{~kg} \mathrm{~N}), 80 \mathrm{~kg}$ mineral N ha ${ }^{-1}(80 \mathrm{~kg} \mathrm{~N}), 175 \mathrm{~kg} \mathrm{~N} \mathrm{ha}^{-1}$ of composts, urban organic waste compost (OWC), green waste compost (GC), farm yard manure compost (MC), sewage sludge compost (SSC), $+\mathrm{N}$ application of $80 \mathrm{~kg} \mathrm{~N} \mathrm{ha}^{-1}$.

Dehydrogenase activity was significantly lower in soils with mineral fertilizer $(80 \mathrm{~kg} \mathrm{~N})$ compared to soil without nitrogen $(0 \mathrm{~kg} \mathrm{~N})$, but no differences were observed between compost treatments with and without nitrogen (Figure 3a). The $\beta$-glucosidase activity (Figure 3b) was significantly higher in OWC, SSC, MC $+\mathrm{N}$ and SSC $+\mathrm{N}$ compared to treatment $0 \mathrm{~kg} \mathrm{~N}$, and similar values were observed with $0 \mathrm{~kg} \mathrm{~N}$ and mineral $(80 \mathrm{~kg} \mathrm{~N})$. Alkaline phosphatase activity (Figure 3c) showed no significant differences between mineral fertilizer $(80 \mathrm{~kg} \mathrm{~N})$ and control and between compost treatment with and without nitrogen. Treatment SSC had significantly higher values in alkaline phosphatase activity than the control and $80 \mathrm{~kg} \mathrm{~N}$ treatment, while no significant differences were observed for urease activity between treatments (Figure 3d). 


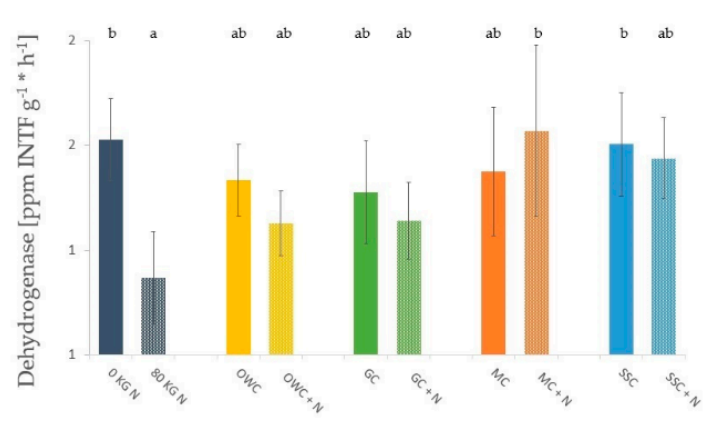

(a)

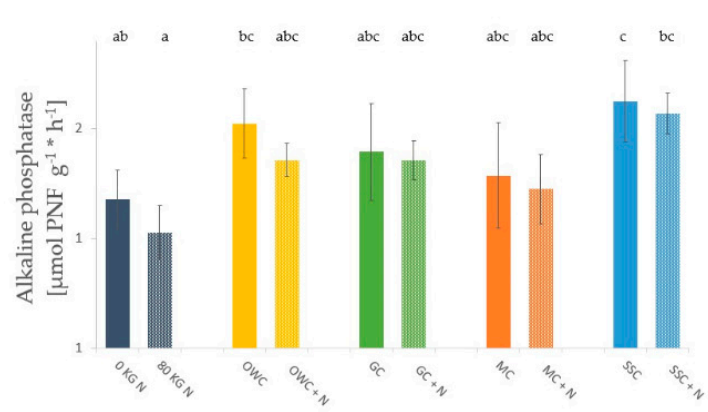

(c)

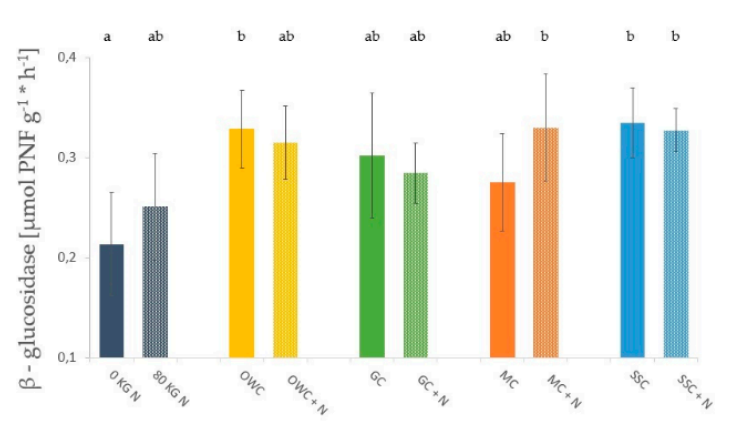

(b)

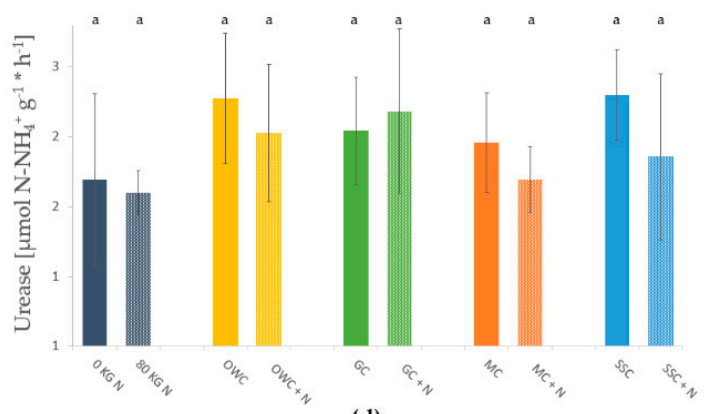

(d)

Figure 3. Soil enzymatic activities (dehydrogenase (a), $\beta$-glucosidase (b), alkaline phosphatase (c) and urease $(\mathbf{d}))($ mean $\pm \mathrm{SD} ; n=4)$. Columns with the same lowercases letters are not significantly different $(p \leq 0.05)$ between treatments. Control $(0 \mathrm{~kg} \mathrm{~N}), 80 \mathrm{~kg}$ mineral N ha ${ }^{-1}(80 \mathrm{~kg} \mathrm{~N}), 175 \mathrm{~kg} \mathrm{~N}^{\circ}$ $\mathrm{ha}^{-1}$ of composts, urban organic waste compost (OWC), green waste compost (GC), farm yard manure compost (MC), sewage sludge compost (SSC), $+\mathrm{N}$ application of $80 \mathrm{~kg} \mathrm{~N} \mathrm{ha}^{-1}$.

\subsection{Soil Microbial Community}

The bacterial/archaeal community compositions differed significantly among $0 \mathrm{~kg} \mathrm{~N}, 80 \mathrm{~kg} \mathrm{~N}$, SSC, OWC, GC and MC (independent from $\mathrm{N}$ addition)-treated soils as calculated from a presence-absence distance matrix (measure $=$ Jaccard, $R_{\text {Adonis }}{ }^{2}=0.396$, $\mathrm{p}_{\text {Adonis }}=0.001$ ) (Figure $4 \mathrm{a}$ ). For the fungal community, such differences were less pronounced $\left(\mathrm{R}_{\text {Adonis }}{ }^{2}=0.14\right.$, $\mathrm{p}_{\text {Adonis }}=0.002$ ) (Figure $4 \mathrm{~b}$ ). The amount of nitrogen added to the soil affected neither fungal nor bacteria/archaeal community composition. Therefore, OTU counts were averaged for soils treated with organic amendments.

The diversity of bacteria/archaea ranged from 10,700 to 11,300 different OTUs in the soils. Proteobacteria was the phylum with the highest number of OTUs in all groups, followed by Actinoand Acidobacteria (Figure 5). Members of Burkholderiaceae (Proteobacteria) and members of the orders Cytophagales (Bacteriodetes), Bacillales (Firmicutes) and Clostridiales (Firmicutes) were found in all soils. The number of OTUs of the fungal community was one order of magnitude lower. For both microbial groups (bacteria/archaea and fungi) no differences in the number of OTUs occurred between treatments. The majority of OTUs were found in all treatments (core OTUs; 1854) (Figure 6a). The addition of $80 \mathrm{~N}$ mineral fertilizer did not impact OTU distributions across treatments (Figure 6b). 


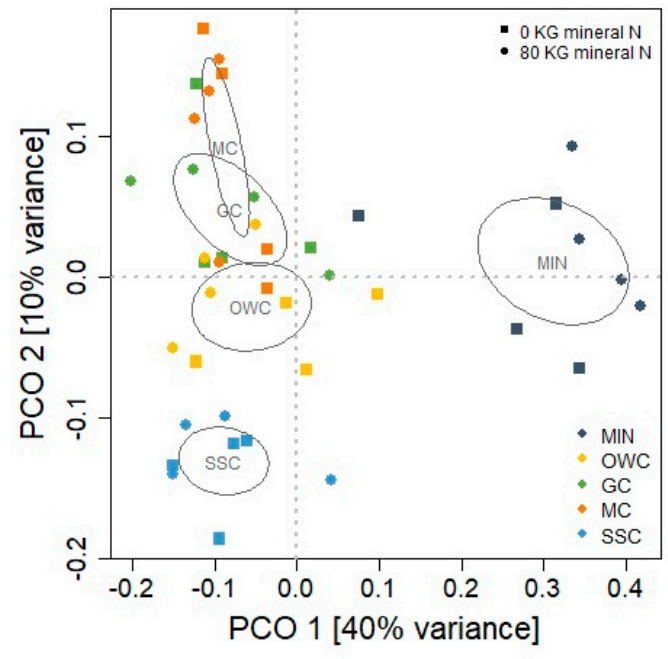

(a)

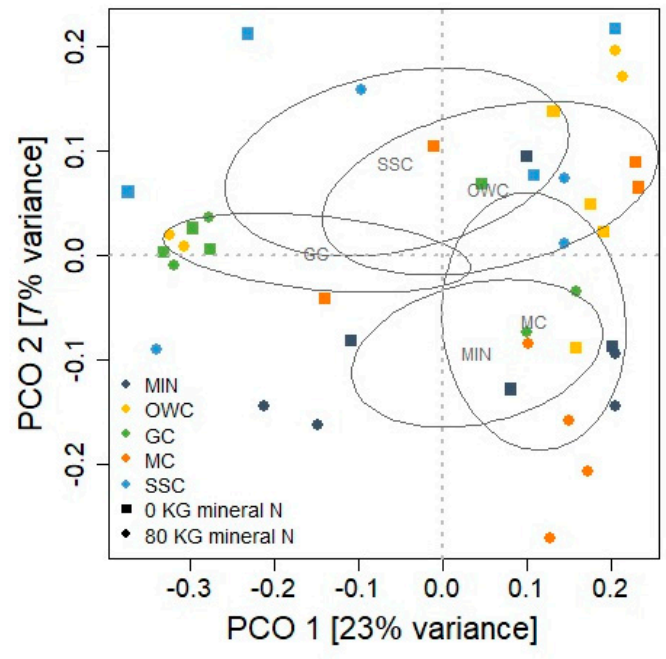

(b)

Figure 4. Principal coordinate analysis of (a) bacterial/archaeal and (b) fungal communities based on 16S and ITS2 genes, respectively. The ordination displays compositional differences between samples based on Jaccard distance. Ellipses indicate the $95 \%$ confidence intervals around centroids.

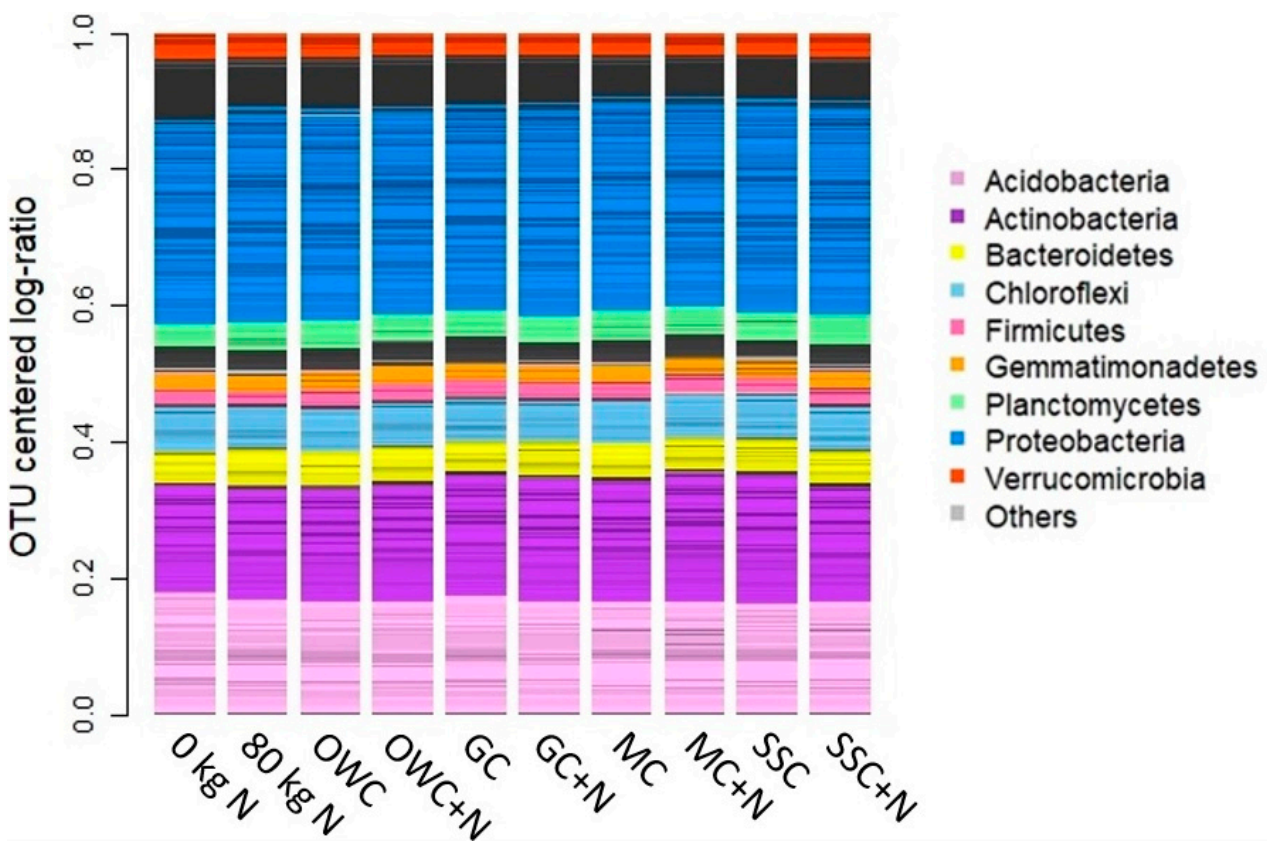

Figure 5. Overview of the taxonomic composition of bacteria in the different treatments. Control $(0 \mathrm{~kg} \mathrm{~N}), 80 \mathrm{~kg}$ mineral $\mathrm{N} \mathrm{ha}^{-1}(80 \mathrm{~kg} \mathrm{~N}), 175 \mathrm{~kg} \mathrm{~N} \mathrm{ha}^{-1}$ of composts, urban organic waste compost (OWC), green waste compost (GC), farm yard manure compost (MC), sewage sludge compost (SSC), + $\mathrm{N}$ application of $80 \mathrm{~kg} \mathrm{~N} \mathrm{ha}^{-1}$.

Interestingly, the control and $80 \mathrm{~kg} \mathrm{~N}$ and OWC treated soils seemed most comparable in Venn analysis, while MC, GC and SSC had a higher number of OTUs detected only in soil samples in those treatments (Figure 6). Soils treated with SSC had more unique OTUs. Those OTUs solely detected in SSC treated soil were taxonomically diverse. Frequently, Paenibacillaceae (Firmicutes), Gemmatimoadaceae (Gemmatimonadetes) and Planctomycetaceae (Planctomycetes) were identified. In addition, Solibacteraceae (Acidobacteria), Cytophageceae (Bacteroidetes), Anaerolinaceae and Caldilineaceae (Chloroflexi) and Bdellovibrionaceae and Haliangiaceae (Proteobacteria) were found. Based on both presence-absence (Figure 5) and relative abundance (Table S2), Blastocatellales 
(Acidobacteria) and Gaiellales (Actinobacteria) seem to be characteristic for SSC treated soils. In GC soils, unique OTUs were annotated as Haliangium and Sorangium (Myxococcales, Proteobacteria) and Planctomycetales (Planctomycetes). In MC soils, unique OTUs often belonged to Clostridia (Firmicutes) and Proteobacteria (order Myxococcales and genus Aquicella). In OWC soils, most unique OTUs belong to Proteobacteria (Myxococcales) and Planctomycetales (Planctomycetes).

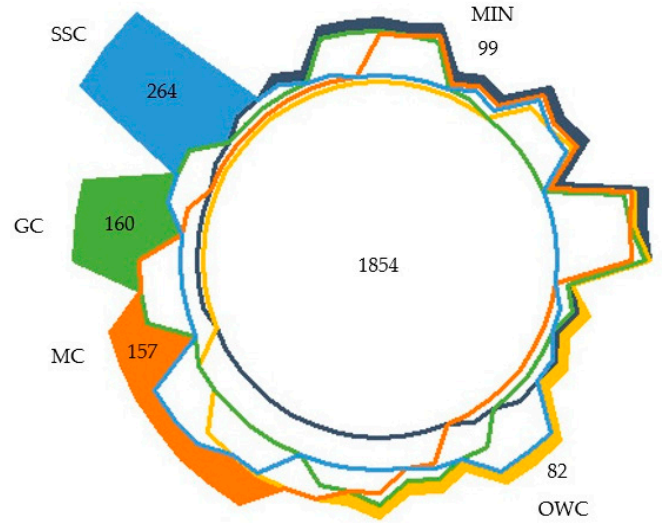

(a)

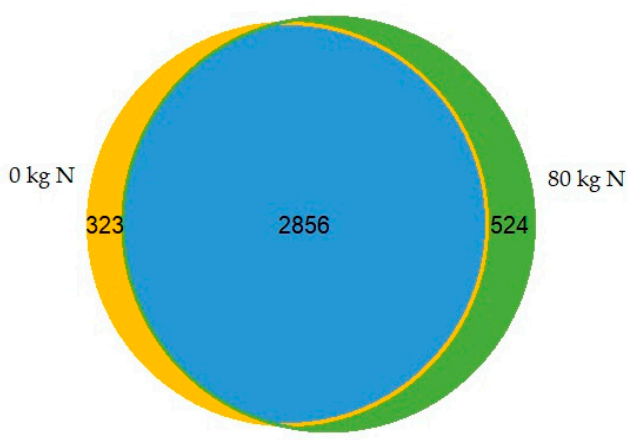

(b)

Figure 6. Venn-diagram of the bacterial/archaeal community regarding treatment factor "compost" (a) and regarding treatment factor "N application" (b). The number in the middle of the circle indicates the number of OTUs shared by all treatments. Numbers near a treatment name show the number of OTUs exclusively found in soils fertilized with this treatment. Use of colors in (a) was done according to Figures 1-3; and in (b) yellow, blue and green code for $0 \mathrm{~kg} \mathrm{~N}$, core and $80 \mathrm{~kg} \mathrm{~N}$, respectively.

\section{Discussion}

\subsection{Crop Yield and Physicochemical Soil Properties}

A 27 year-long maize crop rotation field trial with different fertilization regimes showed that fertilization increased plant yields (Figure 1). In 2018, compost addition, especially GC + N and SSC + $\mathrm{N}$, showed higher maize yields compared to compost addition without mineral $\mathrm{N}$ fertilization, while amending the soil with compost alone showed no significant differences compared to the control and $80 \mathrm{~kg} \mathrm{~N}$. Compost application alone did not increase yield as much as the combination of mineral and organic fertilizers (Figure 1). This is probably due to the slow release of $\mathrm{N}$ from composts, and the growing season might be already over before a sufficient amount of $\mathrm{N}$ becomes available for plants [2]. An additional explanation could be the ability of water extractable humic substances in the soil to enhance root uptake of nitrate, through switching on nitrate transport genes in the roots [19]. Our results suggest that the addition of easily available $\mathrm{N}$ from mineral fertilizers and the more slowly released $\mathrm{N}$ from organic fertilizers enhanced yield compared to control plots. However, compared to the $80 \mathrm{~kg} \mathrm{~N}$ treatment no significant increase of crop yield was detected, indicating that the origin of the compost did not play a crucial role for plant yield and underlining the benefit of additional mineral fertilization.

The use of organic fertilizers led to higher $\mathrm{C}_{\text {org }}$ compared to both the $80 \mathrm{~kg} \mathrm{~N}$-treated soils and the control, which is reasonable, as mineral fertilization does not apply $\mathrm{C}_{\text {org }}$ to the soil. However, only the applications of SSC, GC + N and SSC + N led to significantly higher values. Likely, the incorporation of different organic amendments with and without mineral $\mathrm{N}$ increased the $\mathrm{C}_{\text {org }}$ due to the incorporation of stabilized organic matter [40,41], and also increased the content of $\mathrm{N}_{\text {tot }}$ in soil. This may be attributed to a direct effect of slow-release organic $\mathrm{N}$ derived from the composts [2]. Regarding plant growth, pH and EC especially, but also $\mathrm{Ca}_{\text {excha }}$ and $\mathrm{Na}_{\text {excha }}$, are important parameters to monitor. A high EC indicates a higher salt content, in line with higher amounts of $\mathrm{Ca}_{\text {excha }}$ and $\mathrm{Na}_{\text {excha }}$. This might cause problems in terms of plant growth, especially in combination with high $\mathrm{pH}$ [42]. However, there were 
no negative effects on maize growth in this study, likely because of the generally low salt content (humid climate) and the neutral $\mathrm{pH}$.

Macronutrients in soil are important for plant growth as they are often not present at adequate concentrations. Consequently, amendments with these nutrients seem important for plant yield. The different availabilities of specific macronutrients, however, depend on the specific compost added. Our results suggest that macronutrients are not the main limiting factor for plant yield, as there were no differences between untreated and compost-treated plots compared to plots receiving mineral fertilizer.

In fact, the high amounts of $\mathrm{K}$ and $\mathrm{K}_{\text {excha }}$ and $\mathrm{P}$ in the compost might introduce excess $\mathrm{K}$ and $\mathrm{P}$ after long-term compost application. Thus, the necessity of monitoring soil nutrient supplies is essential to avoid both oversupply and undersupply of nutrients $[2,20]$. Furthermore, the content of heavy metals has to be considered, as they can negatively affect soil health. In our study we used composts with HM concentrations within legally allowed ranges [43]. A rotation in compost fertilization could provide an option to avoid nutrient bottlenecks and HM overload in a similar manner, as crop rotation prevents soil fatigue. Although physicochemical soil properties are proxies for estimating soil fertility, the complexity of soil as an ecosystem makes a clear decision on which compost performed best rather difficult. The picture drawn by the different soil physicochemical properties is necessarily incomplete and despite the significant differences observed in single properties, all compost treatments kept the soil within a plant-supportive range of measured variables. Finally, all compost treatments combined with mineral fertilizers increased plant yield compared to the control.

\subsection{Soil Microbial Biomass and Enzyme Activities}

Our results show that the soils amended with $80 \mathrm{~kg} \mathrm{~N}$ had a significantly lower $C_{\text {mic }}$ value compared to soils amended with composts, except for MC and SSC $+\mathrm{N}$ treated soils. These findings suggest that mixing mineral fertilizer with compost has no negative effect on microbial biomass, which is in agreement with the meta-data-analysis done by Geisseler and Scow [44]. Nevertheless, these authors pointed out that the responses of microbial communities are highly variable and depend on environmental and soil management factors. However, the general trends observed in our study may be overlain by a seasonal effect, since our one-time measurement in autumn 2018 enabled only a snapshot disregarding seasonal cycles. The amount of $C_{m i c}$ also contributes to a higher value of SOM, indicating that organic amendments positively influence SOM build-up. In the same manner, soils amended with $80 \mathrm{~kg} \mathrm{~N}$ showed a significantly lower amount of SOM compared with soil amended with organic fertilizers (MC and SSC), indicating a slower SOM build-up. This effect may be due to stimulated microbial growth through easily available $\mathrm{C}$ and/or to the microorganisms incorporated with the compost [19]. The increase in $C_{\text {mic }}$ is relevant because a high amount of $C_{m i c}$ is considered a good indicator for soil heath compared to $\mathrm{C}_{\text {org }}$, because microorganisms react more rapidly and sensitively on variations in soil management [18]. Higher $C_{\text {mic }}$ implies higher number of metabolically active microorganisms producing enzymes involved in nutrient cycling and contributing to the formation of stable organo-mineral aggregates.

Soil microorganisms express enzymes constitutively, which implies that readily available nutrients lower the rates of enzyme biosynthesis. The dehydrogenase activity can serve as an indicator of overall microbial activity [45].

The activity of $\beta$-glucosidase plays a key role in providing $C$ from SOM for microbes [46]. In line with the higher amount of $C_{\text {mic }}$, we found the highest activity of $\beta$-glucosidase in the OWC, MC $+N$, SSC and SSC + N-amended soils, contrary to the results of Ros et al. [18]. Likely, the bulking agents (e.g., branches and wood chips) of the composts changed over the years, and also lower amounts might have simplified the degradability of OWC and SSC. This variability between composts (even of the same type) and its effect on enzymatic activity highlight the importance of monitoring.

Phosphatases play a key role in the P cycle. Our results confirm those of the previous study of Ros et al. [18]: phosphatase activities were higher in SSC amended soils than in soil solely treated with $80 \mathrm{~kg} \mathrm{~N}$ and the control. This seems reasonable, because sewage sludge contained the highest amount 
of $\mathrm{P}$ (Table 1). On average, phosphatase activities were higher in soils amended with composts than in those amended with mineral fertilizer (Figure 3c). These findings indicate that a sufficient amount of $\mathrm{P}$ was already available in the soils of all treatments, as plant yields were comparable among plots treated with mineral fertilizer and composts, respectively. The application of readily available P also explains the lack of alkaline phosphatase activity in the $80 \mathrm{~kg} N$ treated soils. Here, only total P was measured but not plant and microbially-available fractions [47].

For urease activity no significant differences between treatments and unfertilized samples were detected, which is in accordance with previous findings by Ros et al. [18]. They concluded that the application of composts and mineral fertilizers likely provided sufficient available $\mathrm{N}$, so that no urease activity was induced. This underlines that fertilization, no matter of its origin, provided sufficient $\mathrm{N}$ in this field experiment.

\subsection{Soil Microbial Community}

The bacterial/archaeal community composition of the soils followed the pattern already observed for the soil properties (Figure S1 and Table 2). This was expected, as soil physicochemical properties affect both the prokaryotic community and plant yield and vice versa [48]. Understanding these dynamics is crucial, as they are relevant for nutrient cycling and consequently agriculture. Such knowledge is also highly relevant for the choice of compost, as it does not only affect yield, but the system as a whole. In comparison to the bacteria/archaea, the fungal community did not differ among the treatments (Figure $4 \mathrm{~b}$ ). A probable explanation might be that prokaryotic communities consist of a higher diversity of organisms and adapt faster to changing environmental conditions, such as the high temperatures of $>60{ }^{\circ} \mathrm{C}$ that are reached during the composting process. In contrast, only few fungi are thermophilic or thermotolerant and thus are able to survive the composting process [49].

Microbial diversity is an often discussed topic in ecology, especially related to anthropogenic habitats, such as agricultural ecosystems. Often, high microbial diversity is considered beneficial as it could serve as a kind of "insurance" (e.g., when it comes to severe changes in soil conditions), contributing to a faster soil recovery [50]. In our study we did not detect differences in microbial diversity (Figures 5 and 6a). However, the differences between the microbial communities of the different treatments are striking. Just as two out of many examples, one OTU annotated as Bradyrhizobium (Rhizobiales, Proteobacteria) and one as Nitrospira (Nitrospirales, Nitrospirae), were lower in abundance in SSC samples, compared to other treatments. Another OTU was found in MC-treated soils, which was annotated to the genus of Nitrosomonas, with lower abundance compared to other treatments. As these organisms are involved in N cycling, these findings might highlight that SSC and MC affect $\mathrm{N}$ cycling more compared to the other fertilizers (Table S2). It has to be noted that NGS cannot resolve microbial composition on species, sometimes even at the genus level due to sequence length. In addition, many taxa contain members with different ecological roles. However, even at a higher taxonomic rank, we found many different OTUs annotated as bacterial groups known to be involved in $\mathrm{N}$ cycling, highlighting that different fertilizers differentially affect microbial-related $\mathrm{N}$ cycling in soil. In our study, we also detected Clostridia (Clostridiales, Clostridiaceae), Burkholderia (Burkholderiales, Burkholderiaceae) and Paenibacillaceae (Bacillales). These species who have been repeatedly associated with $\mathrm{N}_{2}$-fixation in maize plants $[13,51]$. The lack of other diazotrophic species might indicate that through fertilization with composts, $\mathrm{N}$ is easily available for the plants. It is known that increased soil mineral $\mathrm{N}$ levels inhibits bacterial N-fixation [52]. Consequently, diazotrophic organisms might be lower in abundance, and therefore, remain undetected. Nonetheless, these probable differences in $\mathrm{N}$ cycling, as observed for SSC, MC and the other treatments, did not influence maize yield. This finding might indicate that easily available $\mathrm{N}$ provided through mineral fertilizers directly enhances plant growth, while microorganisms and high microbial diversity serve the purpose of releasing $\mathrm{N}$ from more recalcitrant plant compounds. 


\section{Conclusions}

Agronomic long-term studies serve the purpose of understanding fertilizer effects after years of application. In the present 27-year crop rotation study with maize at Ritzlhof, Austria, we investigated not only crop yields, but also physicochemical and microbiological soil properties. The addition of GC and SSC in combination with mineral $\mathrm{N}$ fertilizer increased crop yields. We conclude that this was due to the easily available $\mathrm{N}$ from mineral fertilizers in combination with slow-release $\mathrm{N}$-compounds from the compost. The microbial community composition changed depending on the type of compost. However, those differences in the microbial community composition and microbial activity, as found for three enzymes (dehydrogenase, $\beta$-glucosidase and phosphatase), do not seem to influence maize yield. Composts may, thus, well replace mineral fertilizers. However, a combination of compost and additional mineral $\mathrm{N}$ supplementation may be beneficial. We further conclude that SSC is a valuable source of nutrients (particularly P) contributing to increased microbial biomass and activity. However, the utilization of SSC may be limited on the one hand by statutory regulations. On the other hand, the application of SSC might result in excess input of nutrients, such as P. The effects of green waste composts (OWC, GC and MC) were smaller, and the differences among OWC, GC and MC were insignificant. As we hypothesized, compost addition improved microbial activity and biomass over mineral $\mathrm{N}$ fertilization. However, microbial diversity was unaffected.

Supplementary Materials: The following are available online at http://www.mdpi.com/2073-4395/10/11/1664/s1. Figure S1: Principal component analysis (PCoA) of soil properties. Ellipses were drawn at a $95 \%$ confidence interval around group centroids. Table S1: Crop rotation during 1991-2018. Table S2: Differential abundances of bacterial OTUs expressed as centered log-ratio transformed changes as calculated by a generalized linear mixed model. A combination of $80 \mathrm{~kg} \mathrm{~N}$ and the control was used as the reference. Bold values highlight OTUs of high significance. Table S3: Differential abundances of fungal OTUs expressed as centered, log-ratio transformed changes, as calculated by a generalized linear mixed model. A combination of $80 \mathrm{~kg} \mathrm{~N}$ and the control was used as the reference. Bold values highlight OTUs of high significance.

Author Contributions: Conceptualization, F.R.K., H.S., and H.I.; data curation, F.R.K., U.P. and M.P.; formal analysis, F.R.K., M.P.; investigation, F.R.K. and U.P.; methodology, F.R.K., U.P.; project administration, F.R.K.; resources, H.S., T.S., M.R. and H.I.; supervision, H.S., M.R. and H.I.; validation, F.R.K., U.P.; visualization, F.R.K., U.P. and M.P.; Writing-original draft, F.R.K. and U.P.; Writing-review and editing, M.P., H.S., T.S., M.R. and H.I. All authors have read and agreed to the published version of the manuscript.

Funding: This research received no external funding.

Acknowledgments: We want to thank the Austrian Agency for Health and Food Safety for providing results of compost and soil analyses. The provision of an Exzellenzstipendium by the University of Innsbruck is appreciated.

Conflicts of Interest: The authors declare no conflict of interest.

\section{References}

1. Spiegel, H.; Baumgarten, A.; Dersch, G.; Pfundtner, E.; Sandén, T. Impact of mineral P fertilization on trace elements in cropland soils. In Sustainable Agriculture Reviews 29; Lal, R., Francaviglia, R., Eds.; Springer: Cham, Switzerland, 2019; pp. 93-110.

2. Lehtinen, T.; Dersch, G.; Söllinger, J.; Baumgarten, A.; Schlatter, N.; Aichberger, K.; Spiegel, H. Long-term amendment of four different composttypes on a loamy silt Cambisol: Impact on soil organic matter, nutrients and yields. Arch. Agron. Soil Sci. 2017, 63, 663-673. [CrossRef]

3. Amundson, R.; Berhe, A.A.; Hopmans, J.W.; Olson, C.; Sztein, A.E.; Sparks, D.L. Soil and human security in the 21st century. Science 2015, 348, 1261071. [CrossRef] [PubMed]

4. Martínez-Blanco, J.; Lazcano, C.; Christensen, T.H.; Muñoz, P.; Rieradevall, J.; Møller, J.; Antón, A.; Boldrin, A. Compost benefits for agriculture evaluated by life cycle assessment. Review. Agron. Sustain. Dev. 2013, 33, 721-732. [CrossRef]

5. Aguilera, J.; Motavalli, P.; Gonzales, M.A.; Valdivia, C. Initial and residual effects of organic and inorganic amendments on soil properties in a potato-based cropping system in the Bolivian Andean Highlands. Am. J. Exp. Agric. 2012, 2, 641-666. 
6. Shafi, M.; Bakht, J.; Jan, M.; Shah, Z. Soil C and N dynamics and maize (Zea may L.) yield as affected by cropping systems and residue management in North-western Pakistan. Soil Tillage Res. 2007, 94, 520-529. [CrossRef]

7. Tiemann, L.K.; Grandy, A.S.; Atkinson, E.E.; Marin-Spiotta, E.; McDaniel, M.D. Crop rotational diversity enhances belowground communities and functions in an agroecosystem. Ecol. Lett. 2015, 18, 761-771. [CrossRef] [PubMed]

8. Soman, C.; Li, D.; Wander, M.M.; Kent, A.D. Long-term fertilizer and crop-rotation treatments differentially affect soil bacterial community structure. Plant Soil 2017, 413, 145-159. [CrossRef]

9. Rebollido, R.; Martínez, J.; Aguilera, Y.; Melchor, K.; Koerner, I.; Stegmann, R. Microbial populations during composting process of organic fraction of municipal solid waste. Appl. Ecol. Environ. Res. 2008, 6, 61-67. [CrossRef]

10. Ryckeboer, J.; Mergaert, J.; Coosemans, J.; Deprins, K.; Swings, J. Microbiological aspects of biowaste during composting in a monitored compost bin. J. Appl. Microbiol. 2002, 94, 127-137. [CrossRef] [PubMed]

11. Wang, K.; Mao, H.; Li, X. Functional characteristics and influence factors of microbial community in sewage sludge composting with inorganic bulking agent. Bioresour. Technol. 2018, 249, 527-535. [CrossRef]

12. Meada, K.; Hanajima, D.; Toyoda, S.; Yoshida, N.; Morioka, R.; Osada, T. Microbiology of nitrogen cycle in animal manure compost. Microb. Biotechnol. 2011, 4, 700-709. [CrossRef]

13. Chelius, M.K.; Triplett, E.W. The Diversity of archaea and bacteria in association with the roots of Zea Mays L. Microb. Ecol. 2001, 41, 252-263. [CrossRef] [PubMed]

14. Dangi, S.R.; Bañuelos, G.; Buyer, J.S.; Hanson, B.; Gerik, J. Microbial community biomass and structure in saline and non-saline soils associated with salt- and boron-tolerant poplar clones grown for the phytoremediation of selenium. Int. J. Phytoremediation 2018, 20, 129-137. [CrossRef] [PubMed]

15. Sharma, S.K.; Ramesh, A.; Sharma, M.P.; Joshi, O.P.; Govaerts, B.; Steenwerth, K.L. Microbial Community structure and diversity as indicators for evaluating soil quality. In Biodiversity, Biofuels, Agroforestry and Conservation Agriculture; Lichtfouse, E., Ed.; Springer: Dordrecht, The Netherlands, 2010; pp. 317-358.

16. Cui, H.; Yu, S.; Jing, Z.; Haiyan, C.; Long, C.; Dong-Mei, Z. Effect of different grain sizes of hydroxyapatite on soil heavy metal bioavailability and microbial community composition. Agric. Ecosyst. Environ. 2018, 267, 165-173. [CrossRef]

17. Chang, E.; Chung, R.; Tsai, Y. Effect of different application rates of organic fertilizer on soil enzyme activity and microbial population. Soil Sci. Plant Nutr. 2010, 53, 132-140. [CrossRef]

18. Ros, M.; Pascual, J.A.; Garcia, C.; Hernandez, M.T.; Insam, H. Hydrolase activities, microbial biomass and bacterial community in a soil after long-term amendment with different composts. Soil Biol. Biochem. 2006, 38, 3443-3452. [CrossRef]

19. Ros, M.; Klammer, S.; Knapp, B.; Aichberger, K.; Insam, H. Long-term effects of compost amendment of soil on functional and structural diversity and microbial activity. Soil Use Manag. 2006, 22, 209-218. [CrossRef]

20. Spiegel, H.; Mosleitner, T.; Sandén, T.; Zaller, J.G. Effects of two decades of organic and mineral fertilization of arable crops on earthworms and standardized litter decomposition. J. Land Manag. Food Environ. 2018, 69, 17-28. [CrossRef]

21. ÖNORM L1083. Chemical Analysis of Soils-Determination of Acidity. Available online: https://shop.austrian-standards.at/Preview.action;jsessionid=D692D196DF05E2BB172509BDE6B78C4F? preview $=\&$ dokkey=380294\&selectedLocale=de (accessed on 15 October 2020).

22. ÖNORM L1080. Chemical Analyses of Soils-Determination of Organic Carbon by Dry Combustion with and without Consideration of Carbonates. Available online: https://shop.austrian-standards.at/action/de/ public/details/470251/OENORM_L_1080_2013_03_15;jsessionid=00270D8EC9C601EDEF91D5F92544A9C0 (accessed on 15 October 2020).

23. ÖNORM EN16168. Sludge, Treated Biowaste and Soil-Determination of Total Nitrogen Using Dry Combustion Method. Available online: https:/shop.austrian-standards.at/action/de/public/details/ 446011/OENORM_EN_16168_2012_10_01;jsessionid=2F15397C05DF6D81471D3E416159C852 (accessed on 15 October 2020).

24. Tatzber, M.; Schlatter, N.; Baumgartner, A.; Dersch, G.; Körner, R.; Lehtinen, T.; Unger, G.; Mifek, E.; Spiegel, H. $\mathrm{KMnO}_{4}$ determination of active carbon for laboratory routines: Three long-term field experiments in Austria. Soil Res. 2015, 53, 190-204. [CrossRef] 
25. DIN EN 13346. Characterization of Sludges-Determination of Trace Elements and Phosphorus-Aqua Regia Extraction Methods. Available online: https://www.beuth.de/de/norm/din-en-13346/36017256 (accessed on 15 October 2020).

26. Öhlinger, R. Dry Matter and Water Content. In Methods in Soil Biology; Schinner, F., Öhlinger, R., Kandeler, E., Margesin, R., Eds.; Springer: Heidelberg, Germany, 1996; p. 385.

27. Öhlinger, R. Maximum Water-Holding Capacity. In Methods in Soil Biology; Schinner, F., Öhlinger, R., Kandeler, E., Margesin, R., Eds.; Springer: Heidelberg, Germany, 1996; pp. 385-386.

28. Kandeler, E. Ammonium. In Methods in Soil Biology; Schinner, F., Öhlinger, R., Kandeler, E., Margesin, R., Eds.; Springer: Heidelberg, Germany, 1996; pp. 406-408.

29. Kandeler, E. N-Mineralization under waterlogged conditions. In Methods in Soil Biology; Schinner, F., Öhlinger, R., Kandeler, E., Margesin, R., Eds.; Springer: Heidelberg, Germany, 1996; pp. 141-143.

30. ÖNORM L1093. Chemical Analyses of Soils-Determination of Calciumchloride-Extractable Magnesium. Available online: https://shop.austrian-standards.at/action/de/public/details/377609/OENORM_L_1093_ 2010_12_01 (accessed on 15 October 2020).

31. ÖNORM L1086-1. Chemical Analyses of Soils-Extraction of the Effective Exchangeable Cations Ca++, $\mathrm{K}+, \mathrm{Mg}++, \mathrm{Na}+$ and $\mathrm{Al}+++, \mathrm{Fe}+++, \mathrm{Mn}++$ and $\mathrm{H}+$ by Bariumchloride Solution and Determination of the Exchange Capacity. Available online: https:/shop.austrian-standards.at/action/de/public/details/ 518044/OENORM_L_1086-1_2014_03_15;jsessionid=BEF9832C9871F0717DAD63E77B17ABD5 (accessed on 15 October 2020).

32. Tabatabai, M.A.; Bremner, J.M. Use of p-nitrophenyl phosphate for assay of soil phosphatase activity. Soil Biol. Biochem. 1969, 1, 301-307. [CrossRef]

33. Eivazi, F.; Tabatabai, M.A. Glucosidases and galactosidases in soils. Soil Biol. Biochem. 1988, 20, 601-606. [CrossRef]

34. Hupfauf, S.; Etemadi, M.; Insam, H.; Podmirseg, S.M. CoMA—Comparative Microbiome Analysis. 2017. Available online: https:/www.uibk.ac.at/microbiology/services/coma.html (accessed on 27 January 2020).

35. R Development Core Team. R: A Language and Environment for Statistical Computing. R Foundation for Statistical Computing, Vienna, Austria. 2013. ISBN 3-900051-07-0. Available online: http://www.R-project.org (accessed on 27 January 2020).

36. Willis, A.; Martin, B.D.; Trinh, P.; Barger, K.; Bunge, J. Breakaway: Species Richness Estimation and Modeling. R Package Version 4.7.1. 2020. Available online: https://adw96.github.io/breakaway/ (accessed on 20 August 2020).

37. Gloor, G.B.; Macklaim, J.M.; Fernandes, A.D. Displaying variation in large datasets: A visual summary of effect sizes. J. Comput. Graph. Stat. 2016, 25, 971-979. [CrossRef]

38. Swinton, J. Vennerable: Venn and Euler Area-Proportional Diagrams. R Package Version 3.0/r82. 2013. Available online: https://R-Forge.R-project.org/projects/vennerable/ (accessed on 30 April 2020).

39. Van den Boogaart, K.G.; Tolosana-Delgado, R.; Bren, M. Compositions: Compositional Data Analysis. R Package Version 1.40-5. 2020. Available online: https://CRAN.R-project.org/package=compositions (accessed on 20 August 2020).

40. Ros, M.; Hernandez, M.T.; Garcia, C. Soil microbial activity after restoration of a semiarid soil by organic amendments. Soil Biol. Biochem. 2003, 35, 463-469. [CrossRef]

41. Diacono, M.; Montemurro, F. Long-term effects of organic amendments on soil fertility. A review. Agron. Sustain. Dev. 2010, 30, 401-422. [CrossRef]

42. Smith, J.L.; Doran, J.W. Measurement and Use of $\mathrm{pH}$ and Electric Conductivity for Soil Quality Analysis. In Methods for Assessing Soil Quality (SSSA Special Publication 49); Doran, J.W., Jones, A.J., Eds.; Soil Science Society of America: Madison, WI, USA, 1996; pp. 169-185.

43. Gesamte Rechtsvorschrift für Oö. Bodengrenzwerte-Verordnung 2006, Fassung vom 18.05.2020. Available online: https://www.ris.bka.gv.at/GeltendeFassung.wxe?Abfrage=LROO\&Gesetzesnummer=20000409 (accessed on 18 May 2020).

44. Geisseler, D.; Scow, K.M. Long-term effects of mineral fertilizers on soil microorganisms-A review. Soil Biol. Biochem. 2014, 75, 54-63. [CrossRef]

45. Ladd, J.N. Origin and range of enzyme in soil. In Soil Enzyme; Burns, R.G., Ed.; Academic Press: New York, NY, USA, 1978; pp. 51-96.

46. Caldwell, B.A. Enzyme activities as a component of soil biodiversity: A review. Pedobiologia 2005, 49, 637-644. [CrossRef] 
47. Demetz, M.; Insam, H. Phosphorus availability in a forest soil determined with a respiratory assay compared to chemical methods. Geoderma 1999, 89, 259-271. [CrossRef]

48. Tao, J.; Liu, X.; Liang, Y.; Niu, J.; Xiao, Y.; Gu, Y.; Ma, L.; Meng, D.; Zhang, Y.; Huang, W.; et al. Maize growth responses to soil microbes and soil properties after fertilization with different green manures. Appl. Microbiol. Biotechnol. 2017, 101, 1289-1299. [CrossRef]

49. Glassmann, S.I.; Weihe, C.; Li, J.; Albright, M.B.N.; Looby, C.I.; Martiny, A.C.; Treseder, K.K.; Allison, S.D.; Martiny, J.B.H. Decompositions responses to climate depend on microbial community composition. Proc. Natl. Acad. Sci. USA 2018, 115, 11994-11999. [CrossRef] [PubMed]

50. Lynch, J.M.; Benedetti, A.; Insam, H.; Nuti, M.P.; Smalla, K.; Torsvik, V.; Nannipieri, P. Microbial diversity in soil: Ecological theories, the contribution of molecular techniques and the impact of transgenic plants and transgenic microorganisms. Biol. Fertil. Soils 2004, 40, 363-385. [CrossRef]

51. Perin, L.; Martínez-Aguilar, L.; Castro-González, R.; Estrada-de los Santos, P.; Cabellos-Avelar, T.; Guedes, H.V.; Reis, V.M.; Caballero-Mellado, J. Diazotrophic Burkholderia species associated with field-grown maize and sugarcane. Plant Microbiol. 2006, 72, 3103-3110. [CrossRef] [PubMed]

52. Steenhoudt, O.; Vanderleyden, J. Azospirillum, a free-living nitrogen-fixing bacterium closely associated with grasses: Genetic, biochemical and ecological aspects. Fems Microbiol. Rev. 2000, 24, 487-506. [CrossRef] [PubMed]

Publisher's Note: MDPI stays neutral with regard to jurisdictional claims in published maps and institutional affiliations. 